International Journal of Pure and Applied Mathematics

Volume 99 No. 2 2015, 205-208

ISSN: 1311-8080 (printed version); ISSN: 1314-3395 (on-line version)

url: http://www.ijpam.eu

doi: http://dx.doi.org/10.12732/ijpam.v99i2.8

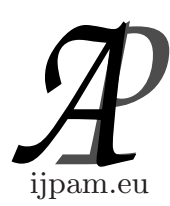

\title{
CYCLICITY ON SOME BK SPACES
}

\author{
B. Yousefi ${ }^{1 \S}$, S.M.A. Musavi ${ }^{2}$ \\ ${ }^{1,2}$ Department of Mathematics \\ Payame Noor University \\ P.O. Box 19395-3697, Tehran, IRAN
}

\begin{abstract}
In this paper, we give some sufficient conditions for cyclicity of adjoint of the multiplication operator acting on a space of formal power series with coefficients in some BK spaces with BK property.
\end{abstract}

AMS Subject Classification: 47B37, 47A25

Key Words: AK space, BK space, invariant subspaces lattice, cyclic vector, shift operator

\section{Introduction}

We write $\omega$ for the set of all complex sequences $x=\left(x_{k}\right)_{k=0}^{\infty}$. Let $\phi, l_{\infty}$ and $c_{0}$ denote the set of all finite, bounded and null sequences. We write $l_{p}=$ $\left\{x \in \omega: \sum_{k=0}^{\infty}\left|x_{k}\right|^{p}<\infty\right\}$ for $1 \leq p<\infty$. By $e^{(n)}\left(n \in N_{0}\right)$, we denote the sequence with $e_{n}^{(n)}=1$ and $e_{k}^{(n)}=0$ whenever $k \neq n$. For any sequence $x=\left(x_{k}\right)_{k=0}^{\infty}$, let $x^{[n]}=\sum_{k=0}^{n} x_{k} e^{(k)}$ be its n-section. Given any subset $F$ of $\omega$, we write $\hat{F}$ for the set of all formal power series $\hat{f}$ with $\hat{f}(z)=\sum_{k=0}^{\infty} f_{k} z^{k}$ where $f=\left(f_{k}\right)_{k=0}^{\infty} \in F$, regardless of whether or not the series converges for any value of z. Let $\hat{M}_{z}: \hat{F} \rightarrow \hat{\omega}$ be defined by $\left(\hat{M}_{z} \hat{f}\right)=\sum_{k=0}^{\infty} f_{k} z^{k+1}$.

A $B K$ space is a Banach sequence space with the property that convergence implies coordinatewise convergence. A $B K$ space $F$ containing $\phi$ is said to have $A K$ if every sequence $f=\left(f_{k}\right)_{k=0}^{\infty} \in F$ has a unique representation $f=$

Received: November 13, 2014

(C) 2015 Academic Publications, Ltd.

${ }^{\S}$ Correspondence author

url: www.acadpubl.eu 
$\sum_{k=0}^{\infty} f_{k} e^{(k)}$, that is $f=\lim _{n \rightarrow \infty} f^{[n]}$; it is said to have $A D$, if $\phi$ is dense in $F$. Given any subset $F$ of $\omega$, the set $F^{\beta}=\left\{a \in \omega: \sum_{k=0}^{\infty} a_{k} f_{k} \quad\right.$ converges for all $f \in$ $F\}$ is called the $\beta$-dual of $F$.

Let $F$ be a normed sequence space and $\hat{F}$ be the space of formal power series with coefficients in $F$ endowed with the norm of $F$. Then $F$ and $\hat{F}$ are norm isomorphic.

We say that a vector $x$ in a Banach space $X$ is a cyclic vector of a bounded operator $A$ on $X$ if $X=\operatorname{span}\left\{A^{n} x: n=0,1,2, \ldots\right\}$. Here $\operatorname{span}\{\cdot\}$ is the closed linear span of the set $\{\cdot\}$. It is convenient and helpful to introduce the notation $<x, x^{*}>$ to stand for $x^{*}(x)$, for $x \in X$ and $x^{*} \in X^{*}$.

In this paper, we study the cyclicity of adjoint of the multiplication operator acting on spaces of formal power series with coefficients in some $B K$ spaces with AK property. For some sources on sequence spaces, see [1-5].

\section{Main Results}

In the main theorem of this paper we give some sufficient conditions for cyclicity of the adjoint of weighted composition operators on BK spaces of formal power series.

Consider $f=\left\{f_{k}\right\}_{k=0}^{\infty}$ and $g=\left\{g_{k}\right\}_{k=0}^{\infty}$ in $\omega$ and let $E \subset \omega$. Define $f g=\left\{f_{k} g_{k}\right\}_{k=0}^{\infty}$ and $g^{-1} \star E=\{f \in \omega: f g \in E\}$. If $\alpha=\left\{\alpha_{k}\right\}_{k=0}^{\infty} \in \omega$ is a given sequence with $\alpha_{k} \neq 0$ for all $k$, then by $1 / \alpha$ we mean $1 / \alpha=\left\{1 / \alpha_{k}\right\}_{k=0}^{\infty}$. Write $\hat{F}(\alpha)=\left(\alpha^{-1} \star F\right)$ for any subset $F$ of $\omega$. From now on we suppose that $\alpha=\left\{\alpha_{k}\right\}_{k=0}^{\infty} \in \omega$ satisfying $\alpha_{0}=1$ and $\alpha_{k} \neq 0$ for all $k \geq 1$.

Lemma 2.1. Let $1<p<\infty$ and $\frac{1}{p}+\frac{1}{q}=1$. Then $\left(l_{p}(\alpha)\right)^{*}=l_{q}\left(\alpha^{-1}\right)$ where $\alpha^{-1}=\left\{\alpha_{n}^{-1}\right\}_{n=0}^{\infty}$.

Proof. Since $l_{p}$ is a BK space with AK property, thus $l_{p}(\alpha)$ is also a $\mathrm{Bk}$ and AK space with respect to the norm $\|f\|_{l_{p}(\alpha)}=\|\alpha f\|_{l_{p}}$ for all $f \in l_{p}(\alpha)$. Furthermore, $l_{p}^{*}$ and $l_{p}^{\beta}$ are isomorphic. Thus we get $\left(\alpha^{-1} \star l_{p}\right)^{\beta}=(1 / \alpha)^{-1} \star l_{p}^{\beta}=$ $(1 / \alpha)^{-1} \star l_{p}^{*}$. Hence $\left(l_{p}(\alpha)\right)^{*}=\left(l_{p}(\alpha)\right)^{\beta}=l_{q}\left(\alpha^{-1}\right)$.

Note that the space $\hat{l}_{p}(\alpha)$ is a reflexive Banach space and the dual of $\hat{l}_{p}(\alpha)$ is $\hat{l}_{q}\left(\alpha^{-1}\right)$. Here for simplicity we use $\|\hat{g}\|$ instead of $\|\hat{g}\|_{\hat{l}_{q}\left(\alpha^{-1}\right)}$.

Theorem 2.2. Let the sequence $\alpha=\left\{\alpha_{n}\right\}$ satisfy $\alpha_{n} \geq 1$ for all $n$ and $f=\left\{f_{n}\right\}_{n=0}^{\infty}$ be a vector in the Banach space $l_{q}\left(\alpha^{-1}\right)$ with infinitely many 
$f_{n} \neq 0$. If $\lim _{n \rightarrow \infty} \sum_{k=n+1}^{\infty}\left|f_{k+m} / f_{n+m}\right|^{q}=0$ for all $m \geq 0$, then $\hat{f}$ is a cyclic vector of $\hat{M}_{z}^{*}$ acting on $\hat{l}_{q}\left(\alpha^{-1}\right)$.

Proof. Put $M=\operatorname{span}\left\{\left(\hat{M}_{z}^{*}\right)^{n} \hat{f}: n=0,1,2, \ldots\right\}$. We have $\left(\hat{M}_{z}^{*}\right)^{n} \hat{f}=$ $\sum_{k=0}^{\infty} f_{k+n} z^{k}$. Now for $f_{n} \neq 0$,

$$
\left\|\left(\hat{M}_{z}^{*}\right)^{n} \hat{f}-f_{n}\right\|^{q}=\left\|\sum_{k=1}^{\infty} f_{k+n} z^{k}\right\|^{q}=\sum_{k=1}^{\infty}\left|f_{k+n}\right|^{q} \alpha_{k}^{-q}=\sum_{k=n+1}^{\infty}\left|f_{k}\right|^{q} \alpha_{k-n}^{-q} .
$$

Therefore

$$
\left\|\frac{\left(\hat{M}_{z}^{*}\right)^{n} \hat{f}}{f_{n}}-1\right\|^{q}=\sum_{k=n+1}^{\infty}\left|\frac{f_{k}}{f_{n}}\right|^{q} \alpha_{k-n}^{-q} \leq \sum_{k=n+1}^{\infty}\left|\frac{f_{k}}{f_{n}}\right|^{q} .
$$

Now by condition of the theorem $\lim _{n}\left(\hat{M}_{z}^{*}\right)^{n} \hat{f} / f_{n}=1$. Thus $1 \in M$ and so for each $n$ we get

$$
\left(\hat{M}_{z}^{*}\right)^{n} \hat{f}-f_{n}=\sum_{k=n+1}^{\infty} f_{k} z^{k-n}=f_{n+1} z+\sum_{k=n+1}^{\infty} f_{k} z^{k-n}
$$

is in $M$. Thus whenever $f_{n+1} \neq 0$, we have $z+\sum_{k=n+2}^{\infty}\left(f_{k} / f_{n+1}\right) z^{k-n} \in M$. Now by induction we prove that $z^{m} \in M$ for all $m$ : let $1, z, \ldots, z^{m-1} \in M$ and note that

$$
\left(\hat{M}_{z}^{*}\right)^{n} \hat{f}=\sum_{k=0}^{m-1} f_{k+n} z^{k}+f_{m+n} z^{m}+\sum_{k=m+1}^{\infty} f_{k+n} z^{k} .
$$

For $f_{m+n} \neq 0$, we have

$$
\begin{aligned}
\left\|\left(\hat{M}_{z}^{*}\right)^{n} \hat{f}=\sum_{k=0}^{m-1} f_{k+n} z^{k} / f_{m+n}-z^{m}\right\|^{q} & =\left\|\sum_{k=m+1}^{\infty} \frac{f_{k+n}}{f_{m+n}} z^{k}\right\|^{q} \\
& =\sum_{k=m+1}^{\infty}\left|\frac{f_{k+n}}{f_{m+n}}\right|^{q} \alpha_{k}^{-q} \\
& \leq \sum_{k=n+1}^{\infty}\left|\frac{f_{k+m}}{f_{m+n}}\right|^{q} .
\end{aligned}
$$

By letting $n \rightarrow \infty$, we conclude that $z^{m} \in M$. Thus indeed $z^{m} \in M$ for all $m$, hence $M=l_{q}\left(\alpha^{-1}\right)$ and so $f$ is a cyclic vector of $\hat{f}$ is a cyclic vector of $\hat{M}_{z}^{*}$. 


\section{References}

[1] L. Bagheri and B. Yousefi, Reflexivity of the shift operator on some BK spaces, Rendiconti Del Circolo Matematico Di Palermo, Volume 2013, DOI 10.1007/s12215-013-0143-5 (2013).

[2] M. Mursaleen and A. K. Noman, On some new difference sequence spaces of non-absolute type, Math. Comput. Modelling, 52 (2010), 603-617.

[3] M. Mursaleen and A. K. Noman, Applications of the Hausdorff measure of noncompactness in some sequence spaces of weighted means, Comput. Math. Appl., 60 (2010), 1245-1258.

[4] E. Malkowsky, Linear operators in certain BK spaces, Bolyai Society Mathematical Studies, 5 (1996), 259-273.

[5] A. Wilansky, Summability through functional analysis, Mathematics Studies 85, North-Holland, 1984. 